\title{
Gott denken - für einen Naturwissenschaftler denkbar?
}

\author{
Bernhard Eylert*
}

\section{Zusammenfassung}

Dieser Beitrag enthält einige Neuerungen in der wissenschaftlichen Auseinandersetzung zwischen naturwissenschaftlicher Erkenntnis, philosophischem Anspruch und mathematischen Möglichkeiten des Existenzbeweises einer gottähnlichen Entität (nach Gödel: God-like being). Nach ausführlicher Diskussion des Ausbleibens der Notwendigkeit einer göttlichen Existenz in der Evolution und des parallel dazu entstehenden Bedürfnisses der Soziologien nach Transzendenz werden die Begriffe Materie, Geist und Denken von verschiedenen philosophischen Ansätzen her beleuchtet, um dann in einem mathematischen Konstrukt einen Gottesgedanken zu formulieren. Aufbauend auf dem Gödel'schen Gottesbeweis von 1970, veröffentlicht 1987 und bewiesen 2013, wird eine weitere Eigenschaft dieser Entität, nämlich die Unendlich-Dimensionalität, mit Hilfe der Topologie gezeigt. Letzterer Beweis ist neu und bisher unveröffentlicht.

\section{Abstract}

This article presents new findings regarding the discussion in science, philosophy and mathematics regarding the proof of the existence of God. The author looks at the theory of evolution and finds no relevance of the existence of God in this area. This is not the case in cultural evolution, as human societies express the wish to address transcendence. The items matter, spirit, and thought are discussed in philosophical terms with the aim of finding a mathematical construct that formulates the idea of God. Drawing on the proof of the existence of God developed by Kurt Gödel in 1970, published in 1987, and finally proven in 2013, this article presents a new predicate of God's entity, the dimension of infinity, in terms of the mathematical field of topology. This proof is new and has not yet been published. The result is mirrored in theological theorems and philosophical views.

\section{Motivation}

Warum befasst sich ein Mathematiker, Naturwissenschaftler und Ingenieur mit der Frage „Gott denken“? Auch einen Naturwissenschaftler lässt die Kernfrage der Philosophie nach dem „Wesen des menschlichen Seins“ oder in der Langfassung „Wie lässt sich die Welt und mit ihr der Mensch als vernünftiges Ganzes in dieser Welt vernünftig verstehen?“ nicht unberührt. Diese Frage(n) zu beantworten, mühen sich die Menschen seit Jahrtausenden und es gibt keine einfachen Antworten. Viele philosophische Schulen haben sich in den letzten 5.000 Jahren gebildet und alle haben unterschiedliche Aspekte aufgedeckt. Diese hier zu erörtern, würde bei Weitem zu weit führen. Dennoch beschäftigt Menschen seit Langem die Frage: Ist Gott für einen Naturwissenschaftler denk- bar? Darüber haben viele große Mathematiker, Naturwissenschaftler und Philosophen intensiv nachgedacht. Auf den einen oder anderen wird in diesem Beitrag noch zu sprechen zu kommen sein. Speziell beim Autor dieses Beitrages hatte sich im Laufe der Zeit ein Gedanke verfestigt, der offensichtlich bisher noch nicht tiefer erörtert wurde: der Beweis der Unendlichkeit Gottes. Drei Dinge haben letztendlich dazu veranlasst, diesen Beitrag zu schreiben, zu veröffentlichen und damit eine Diskussion in Gang zu bringen.

1. In einem Interview der Zeitschrift GEO mit Joseph Henrich aus dem Jahr 2014 heißt es an einer Stelle wörtlich (Hanig 2014): „Wenn man die Entwicklungen von Gesellschaften betrachtet, erscheint Religion wie ein Trick, den die kulturelle Evo- lution sich ausgedacht hat, um uns zur Zusammenarbeit anzuregen."

2. Wenn dieser Gedanke zutrifft, wie passt das Ganze in einen Diskurs über Naturwissenschaft und Gott?

3. Wenn es schon kluge Leute in der Geschichte gegeben hat, die mathematisch einen Gottesbeweis unter wohldefinierten Voraussetzungen führen konnten, kann man daraus auch noch weitere Eigenschaften, z. B. die Unendlichkeit, ableiten?

Es soll zunächst mit einem Missverständnis begonnen werden, das Theologie und Naturwissenschaft über Jahrhunderte entzweite. 
2. Galileo Galilei im Spannungsfeld von Religion und Naturwissenschaft

Naturwissenschaft und Religion erscheinen rückblickend über die Jahrhunderte wie Feuer und Wasser. Das berühmteste Beispiel ist das allgemeinhin kommunizierte Schicksal des italienischen Mathematikers, Physikers und Philosophen Galileo Galilei (15641642), der seine aus heutiger Sicht offensichtlich klaren physikalischen Erkenntnisse widerrufen musste, um dem vermeintlich vernichtenden Urteil der Inquisition zu entgehen. Der deutsche Mathematiker und Astronom Nikolaus Kopernikus (1473-1543) hat aus Furcht vor der Inquisition seine astronomischen Erkenntnisse erst kurz vor seinem Tod veröffentlichen lassen. Ein anderer deutscher Mathematiker und Astronom, Johannes Kepler (1571-1630), wurde von Luther und Calvin wegen seiner astronomischen Bewegungsgesetze, die im Widerspruch zum damals gängigen Weltbild des griechischen Mathematikers, Geographen, Astronomen und Philosophen Ptolemäus ( 100-160 v. Chr.) standen, heftig gescholten und theologisch verurteilt.

Begonnen werden sollte mit dem Erstgenannten. Man muss wissen, dass der Disput Galileis mit den Vertretern der Kirche ursprünglich rein wissenschaftlich war. Mittlerweile ist wissenschaftlich auch geklärt, dass Galileo irrte. Und das aus zwei Gründen.

1. Galilei hatte 1610 ein Heftchen mit dem Titel „Siderius nuncius - Der Sternenbote“ (Galilei \& Blumenberg 2000) herausgebracht und ebendort behauptet, er sei der Erfinder des Fernrohrs.

2. In einer weiteren Abhandlung behauptete Galilei, die Kopernikanische Weltordnung mit Hilfe von Ebbe und Flut beweisen zu können (Galilei 1953).

Das Fernrohr hatte aber der niederländische Optiker Zacharias Janssen (1588-1631) schon 1604 erfunden. Es war zum Zeitpunkt der "Erfindung“ Galileis nördlich der Alpen längst am Markt erhältlich. Ferner wusste zu jener Zeit jeder in der Wissenschaft, dass Ebbe und Flut mit der Bewegung des
Mondes um die Erde und nicht mit der Bewegung der Erde um die Sonne zusammenhingen. Also forderte der seitens der Inquisition verantwortliche Kardinal Robert Bellarmin (1542-1621) von Galileo Galilei im Sinne wahrer philosophischer Wissenschaft, seine im Jahr 1616 aufgestellten Behauptungen als Hypothese und nicht als Wahrheit zu deklarieren. In eigener Selbstüberschätzung kümmerte sich Galileo aber nicht um die Auflage. Im Gegenteil, 1631 publizierte er gegen den Rat der Inquisition und seiner Freunde ein als Dialog aufgebautes Werk „Dialogo sopra i due massimisistemi del mondo“, das die ptolemäische Welt mit der kopernikanischen vergleicht (vgl. Galilei 1953). Papst Urban VIII. (geb. als Maffeo Barberini (1568-1644), Papst der röm.-kath. Kirche), der übrigens ein Freund und Förderer Galileis war, sah jetzt seine Autorität in Gefahr und sich zum Handeln gezwungen. Er zitierte Galilei 1633 vor die Inquisition, um ebendort seine als wissenschaftli-che Wahrheit formulierten Thesen zu widerrufen. Dann nahm das Schicksal den uns bekannten Lauf. Dieses Ereignis ist gepaart mit dem Schicksal Keplers, dem in Tübingen durch den Einfluss von Calvin und Luther die Berufung als Professor zeitlebens versagt blieb. Dieser Tatbestand war auch Urban VIII. bekannt. Er wollte einer Verurteilung Keplers aus Genf mit einer Verurteilung Galileis aus Rom zuvorkommen.

Wissenschaftlich lag Galileo also tatsächlich falsch, denn er konnte zum damaligen Zeitpunkt seine Thesen nicht beweisen. Dafür brauchte es Newtons Gravitationsgesetz in Verbindung mit den Kepler'schen Regeln, und der Vernunft gehorchend, hätte er sie als Hypothese veröffentlichen müssen. Der gedemütigte Galilei, der nie Kerker und Folter seitens der Inquisition erleiden musste, vermarktete seine Niederlage so geschickt, dass alle Welt inn als Opfer der Inquisition sehen sollte. Das brachte inm in Vatikan-kritischen Kreisen zusätzlichen Ruhm ein. Hans Conrad Zander (Zander 2008) und Paul Badde (Badde 2012) haben diese beiden Fälle, Galilei und Kepler, allgemein verständlich in Beiträgen mit zahlreichen Quellen sehr schön zusammenfassend dargestellt.

Nicht nur in der Renaissance wurde in der Wissenschaft mit dem Thema Wahrheit bisweilen sehr eigenwillig umgegangen. Aufklärung und ideologisierte Betrachtung des Falls Galilei, besonders im 19. Jahrhundert, haben zu einer immer größeren Ferne zwischen Naturwissenschaft und Religion, speziell der katholischen Kirche, geführt. Das Thema Wahrheit bzw. der Begriff "wahr" sind in diesem Kontext so bedeutsam, dass sie in einem späteren Kapitel noch ausführlich besprochen werden sollen.

\section{Gott in der Evolution}

Ist Gott in der Evolution gegenwärtig, und wenn ja, wann hat er sich ins (menschliche) Bewusstsein eingebracht? Naturwissenschaftlern geht die Frage nach dem Leben als evolutionärer Prozess sehr nahe. Insbesondere ist aus physikalischer Sicht die Frage nach dem Beginn unseres Universums und aus biologischer Sicht die Frage nach dem Beginn des Lebens von besonderem Interesse. Philosophen, Theologen und Naturwissenschaftler haben unterschiedliche Ansichten zum Begriff "Leben“. Sehr schön hat das Jörg Hacker, Präsident der Leopoldina, in seinem Vortrag an der WWU Münster am 9.6.2015 ausgeführt. Den Aufzeichnungen des Autors zufolge sagte er sinngemäß: „Neben den ethischen und rechtlichen Aspekten beweisen die Genomwissenschaften heute, dass die fundamentalen Lebensprozesse im Zusammenwirken von Genen und Proteinen bestehen. Damit allein kann man Leben aber nicht beschreiben. Verantwortung und Geist sowie die Fähigkeit zur Kommunikation spielen für die Bestimmung des menschlichen Lebens eine weitere wichtige Rolle“ (Hacker 2015).

Eine andere, sehr klare Position bezieht der Physiker und Wissenschaftskabarettist Vince Ebert, der zum Schluss seiner ausgezeichneten Satiresendung "Evolution“ mit dem Fazit aufwartet: „Wir brauchen keinen dreifaltigen Gott, um unsterblich zu sein. Die drei Hauptsätze der Thermodynamik reichen vollkommen aus" (Ebert 2015). Das ist natürlich eine bewusst provokante Äußerung. Biochemisch und physikalisch ist ein Teil der Aussage vollkommen korrekt, denn Menschen bestehen aus etwa $10^{28}$ unterschiedli- 
chen Atomen, und diese gehen nach ihrem Tod andere Verbindungen ein, bleiben aber grundsätzlich bestehen. Mit diesem Tatbestand der atomaren Auflösung und Neubindung nach dem Tode geben sich manche Menschen zufrieden und sehen damit ihre Lebensaufgabe als beendet an. Im Kontext mit Gott verlässt Ebert aber die rein naturwissenschaftliche Ebene. Die Persönlichkeit des Menschen bleibt außen vor. Ein Aspekt, der nicht unbeachtet bleiben darf. Setzt man beides in einen Zusammenhang, muss man die Sache ganzheitlich betrachten. Das aber würde einen neuen Themenkreis öffnen, der in diesem Beitrag nicht beschritten werden soll.

Mit den drei Hauptsätzen der Thermodynamik, mit der Kosmologie, der Quantentheorie oder ganz modern mit der sogenannten String-Theorie kommt man dem Ursprung allen Seins physikalisch nahe. Dieser Zustand wird allgemein hin als Urknall bezeichnet. Die Theorie des Urknalls im Einzelnen zu erörtern, würde an dieser Stelle zu weit führen. Es sei auf die entsprechende Fachliteratur verwiesen. Dennoch sollte man einen Moment beim Urknall verharren. Was weiß man wirklich darüber? Eigentlich sehr wenig. Man hat es hier nicht nur im physikalischen Sinne mit einer Singularität zu tun, sondern besonders auch im mathematischen Sinne mit einem Pol. Physiker wissen nicht, was sich sozusagen ,links auf der Zeitachse', die ja nach der Quantentheorie im Urknall beginnt, abspielt. Für den britischen Astrophysiker und Kosmologen Stephen Hawking beginnt die Welt im Urknall und endet irgendwann im schwarzen Loch. Physikalisch mag das nach der derzeitigen Erkenntnis richtig sein: eine Hypothese. Nach der Relativitätstheorie des deutschen Physikers Albert Einstein (1871-1955) verschwindet mit der Zeit auch der Raum. Demnach stellt sich für Physiker nicht die Frage: Was war vor der Zeit? Dennoch bleibt der denkende Mensch dort nicht stehen. Er fragt sich: Was war vor dem Urknall? Wer oder was hat die Zutaten zur Auslösung des Urknalls (Materiebildung) und damit erst die Möglichkeit des Wirkens der thermodynamischen Gesetze bereitgestellt und ausgelöst? Oder wer oder was hat die ungeheure Energie be- reitgestellt, die sich im Bruchteil einer Sekunde im quantenleeren Raum ausgebreitet hat? Diese Frage ist naturwissenschaftlich derzeit wohl nicht zu beantworten. Sich auf den Zufall allein zurückzuziehen, scheint als Antwort nicht weitgehend genug zu sein. Dennoch stößt man auch hier immer noch nicht auf fruchtbaren Boden für göttliche Notwendigkeit, aber es bietet sich eine Möglichkeit, Gott zu positionieren. Mathematiker haben zwar Lösungen für Probleme der Singularitäten im Spezialgebiet der Funktionentheorie, kennen aber bis heute keine geeignete Funktion oder Funktionsmenge, die den Zustand zum Zeitpunkt des Urknalls vollständig beschreiben könnte. Es wäre vermutlich die Weltformel. Einen anderen Ansatz könnte man in der Theorie des deutschen Mathematikers David Hilbert (1862-1943) finden, mit der die Quantenmechanik mathematisch erklärt werden kann. Dem steht die Unvollständigkeitstheorie des österreichischen Logikers Kurt Gödel entgegen, der bewies, dass ein konsistentes formales System wie die Mathematik nicht vollständig sein kann. Damit war Hilberts Hypothese von der Widerspruchsfreiheit mathematischer Axiome widerlegt. Das ist eine sehr komplexe Materie, für deren Diskussion im Einzelnen an dieser Stelle weder Zeit noch Raum bleiben. Als Zwischenfazit lässt sich aus naturwissenschaftlicher Sicht festhalten: In der Evolution scheint Gott keine Rolle zu spielen. Oder anders ausgedrückt: Zur naturwissenschaftlichen Beschreibung der Evolution wird kein Gott gebraucht, er kommt einfach nicht vor.

\section{Bedeutung der Transzendenz und der Übergang zum Ein-Gott-Kult}

Warum redet alle Welt aber dennoch von Gott, zumindest von Transzendenz? An dieser Stelle kann noch einmal auf das zu Anfang dieser Ausarbeitung zitierte Interview mit Joseph Henrich in der Zeitschrift GEO zurückgekommen werden. Auf die Frage "Aber was treibt uns dann an, komplexe Gesellschaften zu errichten? Was bleibt dann übrig? Ein göttlicher Plan?“ antwortet Henrich: „Ich würde eher sagen: eine Evolution. Wenn man die Entwicklung von Gesellschaften betrachtet, erscheint Religion wie ein
Trick, den die kulturelle Evolution sich ausgedacht hat, um uns zur Zusammenarbeit anzuregen. Anfangs noch nicht: Jäger-und-Sammler-Gesellschaften glauben zwar an übernatürliche Kräfte, aber diese Kräfte moralisieren nicht, sie kümmern sich nicht darum, Menschen für schlechtes Verhalten zu bestrafen. Erst mit dem Auftauchen komplexer Gesellschaften finden wir den Glauben an übernatürliche Kräfte, die bestimmte Werte durchsetzen, welche uns sozialer stimmen" (Hanig 2014).

Unter kulturellen Gesellschaften versteht man in diesem Beitrag Völker mit und ohne staatliche Strukturen, im Gegensatz zu Jäger-und-SammlerGesellschaften, die im Wesentlichen aus Familienverbänden bestehen. Ist Gott also nur ein Gedankenkonstrukt des Menschen, ausgelöst durch die kulturelle Evolution, eine menschengemachte Kreatur zum Zwecke gesellschaftlicher Strukturierung, wenn nicht gar ausschließlich der Disziplinierung der Menschen dienend? Warum erscheint er in der Literatur erst 60-70.000 Jahre später, nachdem sich der Homo sapiens vom Süden Afrikas aus über dessen Ostküste in den Norden aufgemacht hat? Eben doch ein "Hirngespinst“ menschlicher Phantasie?

Dazu muss man noch einmal etwas zurückgehen. Die Einführung des aufrechten Gangs hat beim Menschen erhebliche körperliche und vor allem psychische Veränderungen bewirkt. Sie markiert den Beginn zwischenmenschlicher Beziehungen. In diesem Kontext wurde es notwendig, Familienverbände zu entwickeln. Daraus entstanden die Jäger-und-Sammler-Gesellschaften, aus denen dann, wie bei Ensminger und Henrich (Ensminger \& Henrich 2014) beschrieben, die kulturellen Gesellschaften hervorgingen. Aus archäologischen Forschungen weiß man, dass spätestens in der Steinzeit, dem Magdalénien (ca. 12.000 v. Chr.), die Menschen begannen, den Verstorbenen Grabbeilagen mitzugeben (vgl. Street et al. 1999). Das deutet darauf hin, dass die Menschen spätestens dann begannen, an übernatürliche Kräfte und/oder ein Weiterleben nach dem Tode zu glauben, und das zu einer Zeit, als die 
großen monotheistischen Religionen noch gar nicht existierten. Diese treten erst fast 10.000 Jahre später auf den Plan, darunter die Religion des großen Volks der Juden.

Was kann man daraus lernen? Sicherlich eins: Schon der frühe Homo sapiens hatte, vorsichtig ausgedrückt, einen ,Hang zur Transzendenz'. Neben der reinen Existenz muss da wohl noch was anderes sein, was menschliches Leben bestimmt: der Geist. Und damit ist man wieder bei Jörg Hacker (Hacker 2015), der dieses Element als überaus wesentlich für Leben an sich ansieht. Bis heute entzieht sich den Biowissenschaftlern und Evolutionstheoretikern, zu welchem Zeitpunkt der Geist den Menschen in Besitz genommen hat. Nimmt man das zuvor Gesagte hinzu, muss dies schon sehr früh geschehen sein, möglicherweise im Zusammenhang mit der Einführung des aufrechten Gangs und der sich daraus entwickelnden Psyche. Der Geist ist das Element, was Mensch und Tier wohl am meisten voneinander unterscheidet. Philosophisch ist die Frage nach dem Geist so interessant, dass diesem Thema ein eigenes Kapitel gewidmet werden soll. Dabei soll darüber nachgedacht werden, ob man ,richtig denkt'.

5. Geist und Materie: Was war zuerst da, wer steht höher? - Ein ungelöstes Dilemma

Zurück zu einer der Kernfragen der Philosophie: Geist oder Materie, was war zuerst da? Wer steht höher? Auch diese Frage(n) kann man hier nicht umfassend beantworten, aber einige Aspekte sollen doch vorgestellt werden. Seit mehr als 2.000 Jahren beschäftigen sie die abendländischen Philosophen, und ein allgemeingültiges Verständnis gibt es bis heute nicht.

Zum Ursprung der Materie wurde schon zuvor im Kontext des Urknalls etwas gesagt. Für die alten Griechen war Geist immer ein wesentlicher Bestandteil menschlichen Lebens, der mit dem Tod auch nicht verschwand und als Seele weiterexistierte. Manche Philosophen und Physiker wie Werner Heisenberg (1901-1976) und Karl Friedrich von Weizsäcker
(1912-2007) korrelieren Geist und Materie, wobei die Frage, wer aus wem hervorgeht, heftig umstritten ist. Andere glauben, der Geist sei aus der Materie hervorgegangen (vgl. Livio 2014: 11, Penrose 2002), wieder andere halten die Materie für eine Art Phasenübergang des Geistes zur Materie. Geist und Gedanken gehen eine Symbiose ein. Gedanken brauchen keine Materie, um gedacht zu werden. Der Prozess läuft zwar in Materie (Gehirn, Zellen) ab, Gedanken und Materie bilden aber physikalisch keine Einheit. Man kann diese Funktion mit dem Zusammenspiel von Soft- und Hardware eines Elektronenrechners vergleichen. Ohne den Geist wäre es uns nicht möglich, uns über die Kernfrage der Philosophie "Was ist das Wesen des Seins?" oder "Wie lässt sich die Welt und mit ihr der Mensch als vernünftiges Ganzes in dieser Welt vernünftig verstehen?" Gedanken zu machen. Für Theologen ist der Geist göttlichen Ursprungs, das was Mensch und Gott verbindet.

Der große griechische Philosoph Platon (ca. 428-347 v. Chr.) war der Erste, der dank seiner geistigen Fähigkeiten Philosophie, Mathematik, Physik, Religion, Ethik und Kunst holistisch zu betrachten vermochte. Er hat eine Denkrichtung begründet, die platonische Philosophie, die bis heute viele Anhänger hat. Ein Kernpunkt dieses Denkens ist die Mathematik und ihre Rolle, die sie im geistigen Konstrukt spielt.

1. Für Platon ist die Mathematik ein a priori bestehender Bestandteil des Universums, den es zu, entdecken` gilt (,das Universum ist Mathematik'), oder um es mit dem französischen Mathematiker und Philosophen René Descartes (1596-1650) zu sagen: „Das Universum ist in der Sprache der Mathematik geschrieben. " Diese Auffassung teilen auch die anderen großen Mathematiker und Naturwissenschaftler des 17. Jahrhunderts wie Isaac Newton (16421726) und Galileo Galilei. Für sie wird die Mathematik entdeckt, d. h. sie ist Teil der Schöpfung.

2. Mit der Aufklärung und der Einführung der Logik in das große mathematische Gesamtsystem ändert sich das Bild. Zwar hatte schon der große deutsche Mathematiker und Philosoph Gottfried Wilhelm Leibniz (1646-1716) die beiden Disziplinen Mathematik und Logik miteinander vereint, den Durchbruch dieses Gedankens erlebt man aber erst im 19. Jahrhundert mit den Mathematikern Augustus de Morgan (18061871), George Boole (1815-1864), Gottlob Frege (1848-925) und Giuseppe Peano (1858-1932). Jetzt beginnt das Nachdenken über das Denken. Zwar findet man Ansätze dieser Philosophie schon bei René Descartes mit seinem zentralen Gedanken ,Cogito ergo sum' (,Ich denke, also bin ich'), aber dass Geist und Denken eine Einheit bilden, ist philosophisches Gedankengut des 19. und 20. Jahrhunderts.In dieser Zeit entwickelt sich auch die postplatonische Vorstellung, nach dem die Mathematik erfunden und nicht entdeckt wird. Das bedeutet auch, dass sie nicht a priori war, sondern ihre Wesensmerkmale erfunden werden müssen.

Diese Gegensätze zerreißen fast die Mathematiker und Philosophen bis in unsere Zeit hinein. Als Beispiele für die verschiedenen ,Glaubensrichtungen' seien hier die Mathematiker Godfrey Harold Hardy (1877-1947) (pro platonisch) und Edward Kasner (1878-1955) sowie James Newman (1907-1966) (antiplatonisch) erwähnt. Neuere Veröffentlichungen sprechen für ein Sowohl-als-auch, was als Wigners Mysterium, benannt nach dem ungarischen Physiker Eugene Wigner (1902-1995), in die Literatur (Livio 2014: 296) eingegangen ist. Wigner sieht die Mathematik als ,unbegreifliche Erklärungsmacht ‘ an. So könnte man sagen, die Axiome der Mathematik gehören ins Universum, sind also a priori existent, mussten aber entdeckt werden, während die sich daraus ergebenden Sätze und Konzepte erfunden werden. Hier vielleicht noch zur Erinnerung eine wichtige Definition: Eine Aussage, die einen mathematischen Sachverhalt beschreibt und wahr ist, wird als Satz oder Theorem bezeichnet. 


\section{Wahrheit in Literatur und Religion}

Und damit ist man bei einem ganz entscheidenden Begriffspaar: ,wahr und ,Wahrheit'. Wie schon oben im Fall Galilei angesprochen, hat es mit der Wahrheit schon etwas Besonderes auf sich. Man erinnere sich an die Ringparabel in Lessings "Nathan der Weise“, wo die drei Ringe für die drei monotheistischen Religionen Judentum, Christentum und Islam stehen. Saladin sagt dort: „Von diesen drei Religionen kann doch eine nur die wahre sein“ (Lessing \& Diekhans 2004).

Eine Lehrmeinung, die für Christen unumstößlich ist, lautet:

- Für die Menschen christlichen Glaubens hat sich Gott in Jesus offenbart. Er ist der Messias (Aussage $A$ ), und er ist der Sohn Gottes (Aussage B). So steht es zum Beispiel im Neuen Testament im Johannes-Evangelium.

Dem würden die Menschen jüdischen Glaubens sofort widersprechen, denn für sie hat sich Gott in der Thora offenbart. Dort wird der Messias z. B. bei Jesaja an verschiedenen Stellen zwar angekündigt, sein Kommen steht nach jüdischem Verständnis aber noch aus, und Jesus ist für sie nicht der Messias und schon gar nicht der Sohn Gottes.

Für die Menschen muslimischen Glaubens ist Jesus ein großer Prophet und wird an verschiedenen Stellen des Koran, der muslimischen Offenbarung Gottes, genannt, ist aber nach ihrem Verständnis weder der Messias noch Gottes Sohn.

Jeder widerspricht dem anderen und beruft sich auf seine göttliche Offenbarung. Jede dieser drei Religionen behauptet von sich, aufgrund der jeweiligen Offenbarung im Besitz der Wahrheit zu sein. Ist das möglich? Es wird versucht, die Frage mit Hilfe der Mathematik anzugehen, denn schließlich gibt es dort ein Gebiet, die schon oben angesprochene Logik, die den Begriff ,wahr' als Kernelement beinhaltet.

\section{Wahrheit in der Mathematik}

Unter den Wissenschaften gilt die Mathematik als die einzig exakte Wissenschaft. Sie erfordert ein streng formales Arbeiten. Mathematiker lernen, logisch und strukturell zu denken. Logik und Struktur sind die Grundelemente der Mathematik (Eylert \& Eylert 2014). Man kann nun schauen, was die Aussagenlogik zu bieten hat. Hier geht es um Widerspruchsfreiheit, was der Philosoph , ausgeschlossenen Widerspruch“ nennt und mit ,Vernunft' gleichsetzen würde. Damit hat man die Definition eines wesentlichen Begriffs der o. g. Leitfrage der Philosophie nachgeholt. Die Erkenntnisse der Aussagenlogik sind in unserem Beispiel schon sehr bedeutsam, und deshalb sollen sie an dieser Stelle zur Erinnerung oder Auffrischung kurz wiederholt werden.

Im Gegensatz zur Umgangssprache benutzt die Mathematik eine sehr präzise Sprechweise. Sachverhalte der Realität werden als Aussagen gefasst. Eine solche Aussage kann entweder wahr oder falsch sein. Eine Aussage ist ein ,sinnvolles' sprachliches Gebilde, was der Philosoph wohl ,vernünftig' nennen würde. Da der Mathematiker immer an der Einfachheit und Allgemeingültigkeit interessiert ist, bezeichnet er Aussagen mit lateinischen Großbuchstaben A, $B, C, \ldots$ Nun müssen die Aussagen ja irgendwie miteinander verknüpft werden. Dazu hat er folgende Verknüpfungen eingeführt:

und (in Zeichen $\boldsymbol{\Lambda}$ ),

oder (in Zeichen $\mathbf{V}$ ),

nicht (in Zeichen $\neg$ ),

wenn-dann oder Implikation

(in Zeichen $\Rightarrow$ ) und

genau dann - wenn

(in Zeichen $\Longleftrightarrow$ )

Die Verknüpfung ,oder ' ist genau dann wahr, wenn wenigstens eine Aussage A oder B wahr ist. Die Umgangssprache kennt vorwiegend das Entweder-oder. Mathematisch macht diese Unterscheidung aber keinen Sinn, weshalb man grundsätzlich den einfachen Begriff ,oder' nutzt.

Der Mathematiker fasst diese fünf Verknüpfungen in so genannten Wahr- heitstafeln zusammen. Dabei steht W für wahr und $F$ für falsch. Hier soll nur eine dieser Tafeln zur Sprache kommen: die Implikation oder Folge-Wahrheitstafel, da sie in unserem Kontext eine wichtige Bedeutung spielt:

\begin{tabular}{|c|c|c|}
\hline A & B & A $\Rightarrow$ B \\
\hline W & W & W \\
\hline W & F & F \\
\hline F & W & W \\
\hline F & F & W \\
\hline
\end{tabular}

Die Implikation, aus $A$ folgt $B$, ist nur dann falsch, wenn $A$ wahr und $B$ falsch sind. In allen anderen Fällen ist die Gesamtaussage, aus "A folgt B“ immer wahr. Das wird den unvorbereiteten Leser sicher überraschen, aber man folgt hier ganz streng den Gesetzen der mathematischen Logik. An dieser Stelle sollte man noch einmal in das vorige Kapitel zurückgehen und die dort gemachten theologischen Aussagen anschauen. Dann ist es gar nicht mehr so trivial, die Wahrheit herauszufinden. Mit mathematischen Kenntnissen kann man nicht sicher bestimmen, ob die Kernaussage (christliche Offenbarung) oder eine der beiden oben genannten Erwiderungen (jüdische bzw. muslimische Position), wirklich richtig ist. Denn aus der wahren Gesamtaussage der Implikation lässt sich nicht schließen, ob die Prämisse wahr oder falsch ist. Man ist also ,so klug als wie zuvor'. Folglich muss man einen anderen mathematischen Ansatz wählen, mit dem zu hoffen ist, weiterkommen zu können.

\section{Gödels Gottesbeweis}

Der schon zuvor erwähnte Mathematiker Kurt Gödel hat einen erweiterten logischen Ansatz gewählt. Gödel gilt als der brillante Logiker des 20. Jahrhunderts, der die Logik wesentlich weiterentwickelt hat und auf bedeutenden Arbeiten von Gottfried Wilhelm Leibniz aufbaut. Im Gegensatz zur klassischen Logik spricht man bei dieser Weiterentwicklung von der Modallogik. Sie enthält Operatoren wie möglicherweise gilt (in Zeichen $\diamond$ ) 
und ,notwendigerweise, gilt (in Zeichen []) (vgl. Gödel 1931). Das Ganze wird dann noch komplizierter, wenn man es um Quantoren wie Gott-artig (Leibniz), Gott oder Gott-ähnlich (God-like, Gödel) erweitert.

Mit der Technik der Modallogik hat Gödel 1970 eine Skizze für einen Gottesbeweis geschrieben, der von einem seiner Schüler, Dana Scott, aufgeschrieben und erst nach seinem Tod von Jordan Howard Sobel (Sobel 1987) veröffentlicht wurde. Man nimmt an, dass Gödel sich nicht getraut hat, den Gottesbeweis selbst zu veröffentlichen, da er sich nicht dem Gespött der Leute aussetzen wollte. Günther J. Wirsching hat den Beweis in einem Vortrag in Eichstätt aufbereitet und das Skript im Internet veröffentlicht (Wirsching 2012). Darin ist auch Gödels originale Beweisskizze enthalten:
Setzt man die Gödel'sche Beweisskizze in der Formulierung von Benzmüller und Woltzenlogel Paleo aus der formalen Logik in natürliche Sprache um, dann lautet sie (vgl. Pressestelle der Freien Universität Berlin 2013):

\section{Annahme 1:}

Entweder eine Eigenschaft oder ihre Negation ist positiv.

\section{Annahme 2:}

Eine Eigenschaft, die notwendigerweise durch eine positive Eigenschaft impliziert wird, ist positiv.

\section{Theorem 1:}

Positive Eigenschaften kommen möglicherweise einem existenten Wesen zu.

\section{Definition 1:}

Ein gottähnliches Wesen besitzt alle positiven Eigenschaften.

Gödels Notizen [Transkription von Sobel 1987],

Feb 10, 1970, Ontologischer Beweis:

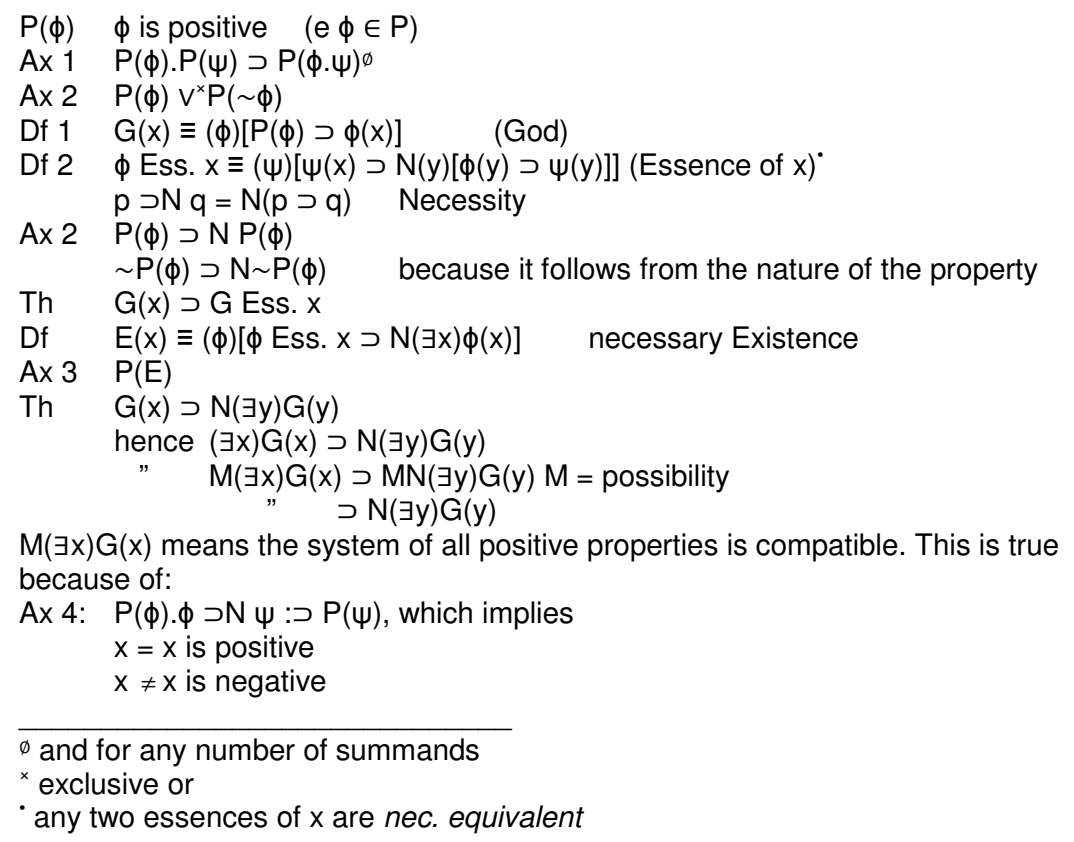

Diese Beweisskizze enthält einige Lücken. Einige Abkürzungen geben Anlass zur Interpretation und werfen Fragen dahin gehend auf, was eigentlich gemeint ist. In den folgenden Jahrzehnten haben sich viele Mathematiker mit dem Thema beschäftigt. Die Mathematiker und KI-Wissenschaftler Christoph Benzmüller von der FU Berlin und Bruno Woltzenlogel Paleo von der TU Wien haben 2013 (Benzmüller \& Woltzenlogel Paleo 2014) die meisten Lücken heben und eine schlüssige Formulierung finden können, auf der dieser Beweis letztendlich abschließend geführt werden konnte.

Annahme 3:

Die Eigenschaft, gottähnlich zu sein, ist positiv.

\section{Schlussfolgerung: Möglicherweise existiert Gott.}

Annahme 4:

Positive Eigenschaften sind

notwendigerweise positiv.

Definition 2:

Eine Eigenschaft ist Kern eines Individuums, falls sie diesem Wesen zukommt und notwendigerweise alle Eigenschaften dieses Wesens impliziert.

Theorem 2:

Gottähnlich zu sein ist Kern jedes gottähnlichen Wesens.

\section{Definition 3:}

Ein Wesen existiert genau dann notwendigerweise, wenn all ihre Kerneigenschaften notwendigerweise in einem existenten Wesen realisiert sind.

Annahme 5:

Notwendigerweise zu existieren ist eine positive Eigenschaft.

\section{Theorem 3:}

Gott existiert notwendigerweise.

Benzmüller und Woltzenlogel Paleo haben mit Hilfe von Computerprogrammen, sog. Theorembeweisern, nachgewiesen, dass der Gödel'sche Gottesbeweis mathematisch in sich konsistent ist und damit auch das Theorem 3 bewiesen werden konnte. Ist damit der Gottesbeweis erbracht? Logisch schon, und dennoch bleiben Fragen offen wie z. B.: "Kann den mathematischen Aussagen eine Bedeutung im realen Leben zugewiesen werden, so wie es Gödel vorschlägt?“ „Welche Bedeutung misst man dem Begriff Gott-ähnlich im täglichen Leben zu?" Oder um es mit Thomas von Aquin sinngemäß auszudrücken: „Stimmen Gegenstand und Verstand miteinander überein?" Beispielsweise müsste der Gottgläubige begründen, warum die Rede von Gott in unserer Welt sinnvoll ist. Andererseits müsste der Atheist nachweisen, warum die verbliebenen Lücken nicht zu schlie- 
ßen sind. Da ist sicher noch nicht das letzte Wort gesprochen, aber Gödels Beweisskizze in Verbindung mit den Beweisen von Benzmüller und Woltzenlogel Paleo sind das Präziseste, was in Sachen ontologischer Gottesbeweis bisher gezeigt wurde.

Nun ist das Lösen alläglicher praktischer Probleme mit Hilfe mathematischer Verfahren das tägliche Geschäft der so genannten ,angewandten Mathematiker, die die Schnittstelle zwischen reiner (mathematischer) Theorie und praktischer Anwendung bilden. Hat der Physiker oder Ingenieur sein Problem klar und deutlich beschrieben, kann der (angewandte) Mathematiker inm das passende mathematische Werkzeug zur Problemlösung zur Verfügung stellen oder auch das Problem für inn lösen. Auch Informatiker sind in erster Linie Praktiker. Sie müssen die zuvor formulierte Problemlösung programmieren und in einen Rechner eingeben, der dann (hoffentlich) die praktische Lösung bringt.

\section{Unendlichkeit Gottes}

Wenn man sich das bisher Gesagte erneut vor Augen führt, dann ist auffällig, dass mathematisch vorgebildete Denker nahezu ausschließlich den logischen Ansatz wählen, wenn es darum geht Gott zu denken. Beobachtet man die Menschen in ihrer Alltagssprache, dann hört man, dass sie den Begriff ,Gott' immer wieder benutzen, sicherlich meist unbewusst, wie in ,Gott sei Dank', ,Um Gottes Willen' oder ,Gott bewahre‘. Wenn Menschen inn aber bewusst benutzen, dann meist im Zusammenhang mit etwas Großem. Die Frage ist nun: Kann man dieses ,Große‘ auch mathematisch bestimmen? Als angewandter Mathematiker schaut der Autor dieses Beitrags in den großen Fundus der Mathematik und da fällt die Topologie auf. Sie beschäftigt sich mit den Eigenschaften ,allgemeiner Räume‘. Ein spezielles Kapitel darin ist die Dimensionstheorie. Fast jeder hat den Begriff ,Dimension“ schon gehört, jeder Studierende der Mathematik und anderer Naturwissenschaften muss sich im Studium damit auseinandersetzen. Die räum- lichen Dimensionen Höhe, Breite, Tiefe sind vielen vertraut; in diesem Raum lebt der Mensch. Mathematiker nennen ihn den dreidimensionalen Raum. Bewegt man sich auf einer Fläche, z. B. einem Blatt Papier, befinden man sich im zweidimensionalen Raum, auch wenn es umgangssprachlich kein Raum mehr ist. Geht man eine Dimension tiefer, hat man es in diesem Bild mit der Gerade zu tun. Wiederum eine Dimension tiefer hat man den Punkt. Er hat keinerlei Ausdehnung - man spricht von der Dimension 0.

Nun konstruiert man daraus ein mathematisches Denkmodell (nach einer Idee von Abbott 1982). Man stelle sich vor, man sei ein Mathematiker der 2. Dimension. Dann könnte man sich nur Flächen vorstellen, Kreise, Quadrate, Rauten etc. Wie erklärt nun der Mathematiker der dritten Dimension dem der zweiten, wie eine Kugel aussieht? Nun, der Mathematiker der 3. Dimension schiebt eine Kugel durch die Fläche und fragt den Mathematiker der 2. Dimension, was er sieht. Er wird ihm ohne Zweifel antworten: ,Ich sehe einen Punkt der immer größer zu einem Kreis mit maximaler Ausdehnung wird und sich dann wieder verkleinert, bis ich nur noch einen Punkt sehe'. Wenn man sich jetzt einen Körper der vierten Dimension vorstellen will, so muss man einen Kunstgriff anwenden. Man geht noch einmal vom einfachen Punkt aus. Fügt man dem einen weiteren Punkt hinzu, so ergibt sich eine Linie, begrenzt durch 2 Punkte. Fügt man nun einen weiteren Punkt in der nächsten Dimension (Ebene) hinzu, gewinnt man ein Dreieck, das durch 3 Linien begrenzt ist. Fügt man wiederum einen Punkt in der nächsten Dimension (Raum) hinzu, so sieht man einen Tetraeder, der durch 4 Dreiecke begrenzt ist. Fügt man nun einen weiteren Punkt in der nächsten, der 4. Dimension hinzu, so sieht man die Konstruktion eines Simplex S4, der durch 5 Tetraeder begrenzt ist. Das könnte man nun fortsetzen und erkennen, dass die nächsthöhere Dimension immer durch $\mathrm{n}+1$ Körper der vorherigen Dimension (n - 1) begrenzt ist. Mathematiker können dies nun ad infinitum fortset- zen und damit weiterarbeiten. Fazit: Man kann immer noch eine Dimension zur vorherigen hinzufügen, d. h. die Dimension strebt mit der natürlichen Zahl $\mathrm{n}$ gegen unendlich, der Mathematiker hat dafür die Schreibweise $n \in \mathbb{N}^{1}, n \rightarrow \infty$.

Auf diesem Denkmodell haben vor rund 100 Jahren die französischen Mathematiker Jules Henri Poincaré (18541912) und Henri Lebesgue (1875-1941) sowie der niederländische Mathematiker Luitzen Brouwer (1881-1966) die Dimensionstheorie aufgebaut und weiterentwickelt. Den Kulminationspunkt dieser Theorie findet man dann in den Arbeiten von David Hilbert, über dessen Theorie schon vorher kurz berichtet wurde. Nun soll der Fokus jedoch auf die früheren Arbeiten von Poincaré, Lebesgue und Brouwer gelegt werden. Sie sind heute ein wenig in Vergessenheit geraten, aber sie lassen sich jetzt sehr gut gebrauchen und mit den Gödel'schen Gedanken verbinden. Ein Wesen, ein Individuum, das alle positiven Eigenschaften beinhaltet, nennt Gödel Gott.

Folgernd aus dem Gödel'schen Beweis setztman $X=G(x)$ mit $X=\left\{x_{1}, x_{2}, \ldots\right\}$ . Die $x_{i}, i \in \mathbb{N}$ repräsentieren die positiven Eigenschaften. Die Mengen $M_{k}$ sind Teilmengen von $X$, mathematisch $M_{k} \subset X . M_{k}$ heißt offene Umgebung von $x_{i}$, wenn $x_{i} \in M_{k}$. O. B. d. $\mathrm{A}^{2}$ sei $x_{1} \in M_{k}, \forall k$. Dann folgt, $X=G(x)$ ist topologischer Raum (vgl. Behnke et al. 1964). Damit kann man ein wichtiges Theorem, das der französische Mathematiker Henri Lebesgue Anfang des letzten Jahrhunderts entwickelt und 1911 veröffentlicht hat, nämlich das Theorem von der Überdeckungsdimension, anwenden. Es besagt: Ein topologischer Raum $X$ ist dann als $\mathrm{n}$-dimensional zu bezeichnen, wenn es ein kleinstes $\mathrm{n}$ gibt, so dass bei jeder hinreichend feinen Überdeckung dieses Raumes durch offene Mengen $M_{n}$ es Punkte $x_{i} \in X$ gibt, die n+1 Mengen des überdeckenden Mengensystems $\mathfrak{M}, M_{n} \in \mathfrak{M}$, $\forall n \in \mathbb{N}$ angehören. Gibt es ein solches $\mathrm{n}$ nicht, so ist der Raum $\infty$-dimensional. (Koepf 2011)

${ }^{1} \in$ bedeutet Element aus; $\mathrm{N}$ bedeutet die Menge der natürlichen Zahlen $1,2,3,4, \ldots{ }^{2}$ O. B. d. A. - mathematisch gebräuchliche Abkürzung für „ohne Beschränkung der Allgemeinheit“ 
Die Anzahl der positiven Eigenschaften ist unendlich, denn wäre sie endlich, dann müsste die nächste Eigenschaft negativ sein und nach Gödel ist dann das Gegenteil positiv - ein Widerspruch. Daraus folgt mit Lebesgue: $X=G(x)$ ist $\infty$-dimensional.

\section{Schlussfolgerung}

Im Sinne der Fragestellung ,Gott denken' konnte man also unter bestimmten mathematischen Voraussetzungen zeigen,

1. dass Gott nicht nur möglicherweise, sondern auch notwendigerweise existiert (Gödel), und darauf aufbauend,

2. dass, wenn Gott existiert, er unendlich dimensional sein muss.

Man muss auch konstatieren, dass es sich bei Gödels Beweis nicht um einen genuin mathematischen Beweis handelt, denn er greift in den Voraussetzungen mehrfach auf nicht mathematische Erkenntnisse (z.B. „positive Eigenschaften“) zurück. Aber schon Platon sagt: "Das Gute scheint notwendig." Dass Gott unendlich sei und weder Anfang noch Ende habe, ist wie oben gesagt - im Theismus nicht neu. Man findet eine ausführliche Diskussion dieser These u. a. in „Proslogion Seu Alloquium De Die Existentia“ des großen englischen Theologen und Philosophen Anselm von Canterbury (1033-1109). Neu ist nur, Gott mit Hilfe der Modallogik zu beweisen und darauf aufbauend ihm mit der Dimensionstheorie eine Topologie unendlicher Dimension zuzuordnen.

Daraus lassen sich zwei Schlussfolgerungen ziehen:

1. Mathematisch-naturwissenschaftliche Überlegungen müssen nicht notwendigerweise atheistisch enden. Der rationale Theismus macht durchaus Sinn.

2. Keine Wissenschaft soll sich über die andere erheben, weder die Mathematik über die Religion, die Religion über die Philosophie, die Philosophie über die Mathematik und im Ringschluss jede über die jeweils andere.
Diese Gedanken passen schlüssig in die Argumentation eines realen Theismus, wie er z. B. bei Holm Tetens (Tetens 2015) in "Ein eschatologisches ,Eperimentum Crucis“" vorgetragen wird. Damit ist noch nichts über die Kernthese des theistischen Idealismus von der Auferstehung der Toten, von Gericht, Vergebung und Versöhnung der Menschen gesagt, auch nicht über das Verhältnis von Gott zu den Menschen und umgekehrt oder über die verschiedenen Beschreibungen Gottes im Alten Testament oder die 99 Namen Gottes bei den Muslimen. Das war und ist nicht das Ziel dieser Arbeit, und es ist von der Mathematik nach Ansicht des Autors auch nicht zu leisten. Man kann sie in diesem mathematischen Modell nicht abbilden. Das gilt insbesondere auch für die Trinität (ein Gott in drei Personen), die von Matthias Haudel in seinem Buch "Gotteslehre" (Haudel 2015) aus dem historisch-philosophischen Kontext heraus sehr verständlich beschrieben wird. Es kommt bei diesem Beitrag, insbesondere in Verbindung mit dem neuen Unendlichkeitsbeweis Gottes, darauf an, weitere, u. U. auch neue Diskussionen zwischen den Vertretern der angesprochenen Wissenschaften anzuregen.

\section{LITERATUR}

Abbott EA (1982) Flächenland. Reihe reprinta historica didactica, Bd. 5. Franzbecker - Didaktischer Dienst, Bad Salzdetfurth über Hildesheim. ISBN: 3881200207

Badde P (2012) Galileo Galilei - übereifrig, skrupellos, verwildert. Die Welt

Behnke H, Remmert R, Steiner HG, Tietz H (eds) (1964) Mathematik 1. Das Fischer Lexikon, Frankfurt (Main)

Benzmüller C, Woltzenlogel Paleo B (2014) Formalization, Mechanization and Automation of Gödel's Proof of God's Existence. Frontiers in Artificial Intelligence and Applications(263)

Ebert V (2015) Evolution. 3sat festival 2014

Ensminger JE, Henrich J (eds) (2014) Experimenting with social norms. Fairness and punishment in cross-cultural perspective. Russell Sage Foundation, New York NY. ISBN: 978-1-61044-840-6

Eylert B, Eylert D (2014) Kryptologische Grundlagen. In Eylert B (ed) Informationssicherheit. Steganographie, Kryptologie, Organisation und Recht. Wildau Verlag, Wildau, ISBN: 978-3-945560-01-3, pp 18-38

Galilei G (1953) Dialogue on the great world systems. In the Salusbury translation. Rev., annotated, and with an introd. by Giorgio de Santillana

Galilei G, Blumenberg H (2000) Sidereus Nincius. (Nachricht von neuen Sternen). Taschenbuch Wissenschaft, vol 337. Suhrkamp, Frankfurt am Main. ISBN: 9783518279373
Gödel K (1931) Über formal unentscheidbare Sätze der 'Principia Mathematica' und verwandter Systeme. Monatshefte für Mathematik und Physik(38):173-198

Hacker J (2015) Was ist Leben - Reflexionen über eine alte Frage im Zeitalter der Genomwissenschaften. Bernhard Rensch Vorlesung

Hanig F (2014) Wieso wir verschieden ticken. Im Gespräch mit Joseph Henrich. Geo Magazin(5):41-44

Haudel M (2015) Gotteslehre. Die Bedeutung der Trinitätslehre für Theologie, Kirche und Welt, 1. Aufl. UTB, vol 4292. Vandenhoeck \& Ruprecht, Göttingen. ISBN: 978-3-8252-4292-3

Koepf W (2011) 1911: Der mathematische Dimensionsbegriff. http://www.mathematik.de/ger/information/ kalenderblatt/dimensionsbegriff/dimensionsbegriff. html

Lessing GE, Diekhans J (eds) (2004) Nathan der Weise. Ein dramatisches Gedicht in fünf Aufzügen, 6th edn. Einfach deutsch. Schöningh, Paderborn. ISBN: 978-314-022287-7

Livio M (2014) Ist Gott ein Mathematiker? Warum das Buch der Natur in der Sprache der Mathematik geschrieben ist, Ungekürzte Taschenbuchausg. dtv, vo 34800. Dt. Taschenbuch-Verl., München. ISBN: 978-3423-34800-3

Penrose R (2002) Computerdenken. Die Debatte um künstliche Intelligenz, Bewußtsein und die Gesetze der Physik. Spektrum Akad. Verl., Heidelberg u. a. ISBN: 3-8274-1332-X

Pressestelle der Freien Universität Berlin (2013) Gödels "Gottesbeweis" bestätigt. Wissenschaftler der Freien Universität und der TU Wien überprüfen Argumentationskette des österreichischen Mathematikers mit Hilfe von Computern

Sobel JH (1987) Gödel's ontological proof. In: Thomson JJ (ed) On being and saying. Essays for Richard Cartwright. MIT Press, Cambridge Mass. u. a., ISBN: 0-26220063-5, pp 241-261

Street M, Baales M, Jöris O (1999) Beiträge zur Chronologie archäologischer Fundstellen des letzten Glazials im nördlichen Rheinland. In: Becker-Haumann R (ed) Terrestrische Quartärgeologie. Logabook, Köln, ISBN: 3-934346-03-0, pp 426-465

Tetens H (2015) Gott denken. Ein Versuch über rationale Theologie. Was bedeutet das alles?, Nr. 19295. Reclam, Stuttgart. ISBN: 978-3-15-019295-5

Wirsching GJ (2012) Der Gödelsche Gottesbeweis. Eichstätter theologisch-mathematisches Kolloquium

Zander HC (2008) Warum die Inquisition im Fall Galilei Recht hatte. Die Welt

\section{AUTOREN}

Prof. Dr. Bernhard Eylert (Emeritus) Technische Hochschule Wildau

E-Mail für Korrespondenz: bernhard.eylert@th-wildau.de

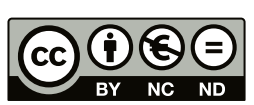

